\title{
Bienvenue et bon travail!
}

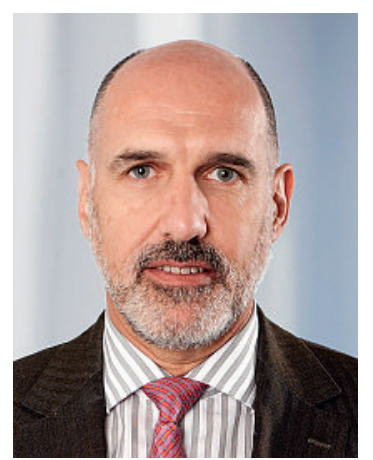

Fin octobre, avec le départ de Pascal Couchepin, une ère politique s'est achevée pendant laquelle les fronts s'étaient progressivement durcis entre les autorités et le corps médical. Le ministre de la santé démissionnaire avait réussi l'exploit non seulement de bloquer toute évolution du système de santé, mais aussi de confirmer tous les présupposés, tous les préjugés possibles du corps médical envers le gouvernement.

Avec Didier Burkhalter commence une époque nouvelle, dont nous espérons beaucoup.
- la démographie des professionnels de la santé, et la démographie médicale en particulier, est une question explosive, tant à cause du gigantesque recours à l'immigration dont nous avons besoin qu'à cause des problèmes de répartition territoriale; le mode de vie de notre société est actuellement centré sur les villes, mais les soins (notamment d'urgence) doivent évidemment rester aussi disponibles en périphérie.

Il nous appartiendra donc, à nous comme aux autres partenaires du système de santé et aux autorités politiques et administratives, de savoir entrer dans un fonctionnement constructif, qui ne soit pas marqué plus que de raison par l'histoire récente et par les tombereaux de conflits qu'elle laisse derrière elle. Il nous faut une époque nouvelle.

\section{Il est clair que personne n'a les moyens de faire avancer sa cause seul. Nous devons donc tous négocier, c'est-à-dire proposer, marchander, donner et recevoir}

Les premiers contacts avec lui laissent entrevoir la possibilité d'un dialogue qui permette de développer et d'adapter le système de santé ensemble, avec le gouvernement et les partenaires concernés.

C'est indispensable, et nous nous réjouissons de voir cette ouverture se confirmer dans les temps qui viennent.

\section{Notre système de santé doit évoluer, il doit $s^{\prime}$ adapter. On ne peut simplement pas geler les choses telles qu'elles sont}

C'est indispensable parce qu'effectivement, vous le savez bien, notre système de santé doit évoluer, il doit s'adapter. On ne peut simplement pas geler les choses telles qu'elles sont - tous les protagonistes, tous, ont tout à gagner à un système qui reste en résonance avec les impératifs de son temps. Pour ne citer que trois exemples:

- le mode de financement que nous connaissons arrive clairement à ses limites; il devient si insupportable qu'il va menacer à terme la qualité des soins;

- la compensation des risques doit enfin permettre un fonctionnement normal du système, alors qu'on voit actuellement combien les lois du marché, de la concurrence, pervertissent le système de santé;
Il ne s'agit pas là d'être naïf ou candide; des désaccords profonds subsisteront, des affrontements se produiront toujours, et un système de santé idéal ne va pas éclore par magie.

Mais il est clair aussi que personne n'a les moyens de faire avancer sa cause seul - même le parlement ne le peut pas, comme l'a montré la tentative un peu échevelée de mesures urgentes «interpartis» l'été dernier. La seule chose qu'on puisse faire plus ou moins seul, dans le contexte politique actuel, c'est de bloquer toute évolution ...

Nous devons donc tous négocier, c'est-à-dire proposer, marchander, donner et recevoir.

Et dans toute négociation, il y a des points intangibles, des «no go's»; il y a des questions négociables; et il y a les «dénominateurs communs» qu'il s'agit d'exploiter pour avancer. Les contacts que nous avons actuellement avec les divers partenaires du système de santé, les assureurs, les cantons et les hôpitaux, ainsi qu'avec le nouveau chef du Département de l'intérieur, montrent que ces dénominateurs communs existent.

Nous aurons à en reparler, assurément, dans ces colonnes, et je m'en réjouis.

Dr Jacques de Haller, Président de la FMH 